\title{
THE USE OF TECHNOLOGY BLUEPRINTS AS A FORESIGHT INSTRUMENT FROM A PRACTITIONER'S POINT OF VIEW
}

\author{
Robert E. Samuel, Widener University, robert.samuel@ieee.org
}

\begin{abstract}
Practitioners often use technology blueprints to articulate future technology planning and strategy. In spite of the proven corporate usefulness, few research studies have attempted to align technology blueprints to the field of technology foresight. This study of a Fortune 500 enterprise technology architecture team examines technology blueprints as a technology foresight instrument. Findings indicate that technology blueprints seem to have similar attributes to other foresight activities based on capabilities and frequency of foresight methods used. However, the foresight capabilities that are most often selected might not align the practitioner's decision-making style.
\end{abstract}

Keywords: Technology Foresight, Enterprise Architecture, Management of Technology, Technology Blueprints, Decision-making, Adaption-Innovation Theory

\section{INTRODUCTION}

We live in a rapidly changing world where varying factors affect both the technologies we use and the purpose they serve. Due to the lengthy lead times demanded by many industries to adopt technology, it is essential to create a view of the future based on complex and uncertain information. During this period of deliberation, technology is advancing and products, using current technologies to satisfy today's customer needs, may become technically obsolete [5]. We have witnessed this phenomenon in recent times with digital photography displacing film, online websites eliminating the need for print encyclopedias, and Voice over IP transforming the telecommunications industry [8]. Clements and Northrop [3, p. 328] in their book, Software Product Lines: Practices and Patterns, elaborates on this theme by stating that technology forecasting "spots emerging standards, allowing an organization to position itself early to lead, or at least to react with agility. It reduces risk with respect to innovations, provides the basis for planning and directing investments in research and development areas, and helps set the direction of product migration." As proven by large corporations, government agencies, and major universities, technology planning aims to improve the quality and timeliness of key technical decision-making during activities such as responding to proposals, funding of government grants, and planning strategic corporate direction. It is not about finding the 'right' decision, but rather the approach to assist leaders in making better decisions.

In the 1970's, forecasting research focused on methods and techniques for predicting the future mainly using past data $[8,12]$. As the scope of research expanded to include information gathering, assessment and interpretation, and decision making, the term foresight emerged [8]. Technology foresight is used throughout the world as a means to derive intuition, experience, knowledge, and skills of technical domain experts for the purpose strategic scheduling and decision-making [13]. For the purpose of this article, technology foresight is defined as [2, p. 52]:

"a one-time activity or an ongoing process within the company. It can be done at the single business, group, whole company, or even the industry level. It is the background for forecasting, strategic planning, $R \& D$ strategy, and decision-making when linked to the business. Most important, it enables the identification and characterization, in business terms, of major new technology opportunities for the enterprise."

Additionally, science and technology foresight is an "emergent and convergent field of learning and strategy, policy and action" [10, p. 92]. 


\section{Issues in Information Systems}

Volume 13, Issue 2, pp. 219-225, 2012

Smith and Saritas [10, p. 82] states that science and technology foresight "involves systematic attempts to look into the longer-term future of science and technology, and their potential impacts on society, with a view to identifying the emerging change factors, and the source areas of scientific research and technological development likely to influence change and yield the greatest economic, environmental and social benefits". They [10] further classify foresight methods into systemic foresight process phases of understanding, synthesis \& models, analysis \& selection, transformation, and actions. Within each phase, several methods are available to the practitioner [10]:

- Understanding phase contains methods such as scanning, bibliometrics, crowd sourcing, literature review, interviews, trends/driver indicators, and system mapping.

- Synthesis \& Models phase contains gaming, scenario planning, wild card weak signals, network analysis, agent-based modeling, and dynamic variable simulations.

- Analysis \& Selection phase contains SWOT analysis, multi-criteria analysis, computerized scenario comparisons, prioritization/DELPHI, scoring voting/rating, and benefit/cost/risk analysis.

- Transformation phase contains backcasting, road mapping, relevance trees, logic charts, technology emergence pathways, and strategic planning.

- Action phase contains priority lists, critical/key technologies, research \& development planning, action planning, operational planning, and impact assessment.

Popper [7] also identifies the five phases of technology foresight with slightly different terms:

- Pre-foresight or Scoping phase - early process decisions established

- Recruitment phase - selection and enrollment of key individuals with knowledge and expertise

- Generation phase - prospective knowledge and visions are created

- Action phase - reaching commitment on decisions with implementation plans

- Renewal phase - understanding the opportunities and threats identified

Comparing Smith and Saritas' phases with Popper's phases, it seems the phases themselves indicate consistency with the overall process.

\section{Technology Blueprints}

Practitioners often use technology blueprints as an enterprise architecture process and set of deliverables to articulate future technology planning and strategy. Technology blueprints are common strategy-based artifacts used in industry by enterprise architecture practitioners known as "technology architects" [9, 11]. In spite of proven corporate usefulness, however, few research studies have attempted to align technology blueprints to the field of technology foresight. Like technology foresight, technology blueprints align business value with the adoption and lifecycle management of technology. As Carlson [2] states, companies that regard technology as a critical element for business success require the core discipline to manage present and emerging technologies to enhance their competitive position. Many Fortune 500 firms use technology blueprints as a means to capture, elaborate, and disseminate their techno-social business strategy $[9,1]$.

In James Butler's [1] book, Technology Blueprints: Technology Foundations for High Performance Companies, he defines technology blueprints as structured collections of patterns applied to the technology aspects of the business operations domain. These patterns are abstracted and recurring solutions to common business opportunities. Butler [1] identifies the structural elements of technology blueprints to include:

- Classification - is the taxonomy by which problem spaces are assigned for categorization and communication.

- Objectives - the desired socio-technical and business outcomes of the blueprint.

- Applicability - provides the scope and context of the problem area being addressed.

- Change agents - are the responsible individuals, teams, and/or vendors suited to implement the blueprint.

- Key points - highlight the primary themes and synopsis of significant elements.

- Foundation - elaborates the details of the problem, the fundamental capabilities, and the rational for business value. 


\section{Issues in Information Systems}

Volume 13, Issue 2, pp. 219-225, 2012

These technology blueprint structural elements are reflected in the field of technology foresight. The purpose of this study is to determine the degree of alignment between technology blueprints and technology foresight.

\section{RESEARCH METHODOLOGY}

Popper [7] studied 886 international foresight studies from the European Foresight Monitoring Network and Strategic Euro-Latin Foresight Research and University Learning Exchange to understand which foresight styles of forward-looking practices and methods were employed. He researched the two fundamental attributes of foresight methods being nature (qualitative, quantitative, or semi-quantitative) and capabilities (evidence, experience, interaction, or creativity). He mapped the various foresight methods to the three nature categories:

- Qualitative - examples include literature review, surveys, and SWOT Analysis

- Quantitative - examples include simulation, trend extrapolation, and bibliometrics

- Semi-quantitative - examples include structural analysis, Delphi, and stakeholder mapping

Additionally, 25 foresight methods were mapped to the capabilities attributes defined as [7, p. 65]:

- "Creativity refers to the mixture of original and imaginative thinking";

- "Expertise refers to the skills and knowledge of individuals in a particular are or subject";

- "Interaction recognizes that expertise often gains considerably from being brought together and challenged to articulate with other expertise";

- "Evidence recognizes that it is important to attempt too explain and/or forecast a particular phenomenon with the support of reliable documentation and means of analysis."

Popper [7, p. 84] concluded, "the findings have revealed that foresight practices are under exploiting existing methods based on creativity and interaction." He also concludes that six methods on average, without extreme cases, were used during foresight exercises. Potter reflects on this observation by indicating that both the variation and diversity of methods is high. Of these methods, he discovered that the nature of qualitative was the most common. The top three methods and the total of ten out of 14 methods were qualitative.

The purpose of this study is to investigate how technology architects, through the creation of technology blueprints, are consistent with the observations from previous foresight research. This study explores following research questions:

RQ1: Do technology blueprints reflect attributes of a technology foresight instrument through foresight practices?

RQ2: Which foresight method attributes do technology architects prefer?

RQ3: Does the participant's decision-making style align to the foresight methods attributes?

This study examined an online survey which was administered to a U.S. based Fortune 500 enterprise technology architecture team in April 2012. The survey questionnaire consisted of five questions that covered the participant's gender, the participant's work duration as a technology architect, the typical number of foresight methods used, the frequency of foresight capabilities, and a self-assessment of decision-making. All participants were notified that the survey was strictly voluntary and would not impact their work performance management reviews. Additionally, a review of the technology blueprint deliverables and overall process was conducted. Ten completed technology blueprints were extensively reviewed for the use of foresight methods and practices.

\section{RESULTS}

Even for large corporations, enterprise technology architecture teams traditionally have a small number of staff [9]. For this study, the firm has ten technology architects within the technology architect role and nine participated in the survey. The years of experience as a technology architect demographic of the sample population is shown in Figure 


\section{Issues in Information Systems}

Volume 13, Issue 2, pp. 219-225, 2012

1. Since this core discipline is a relatively new job position, the years of experience aligns with expectations. The sample population had more males $(66.7 \%)$ than females $(33.3 \%)$.

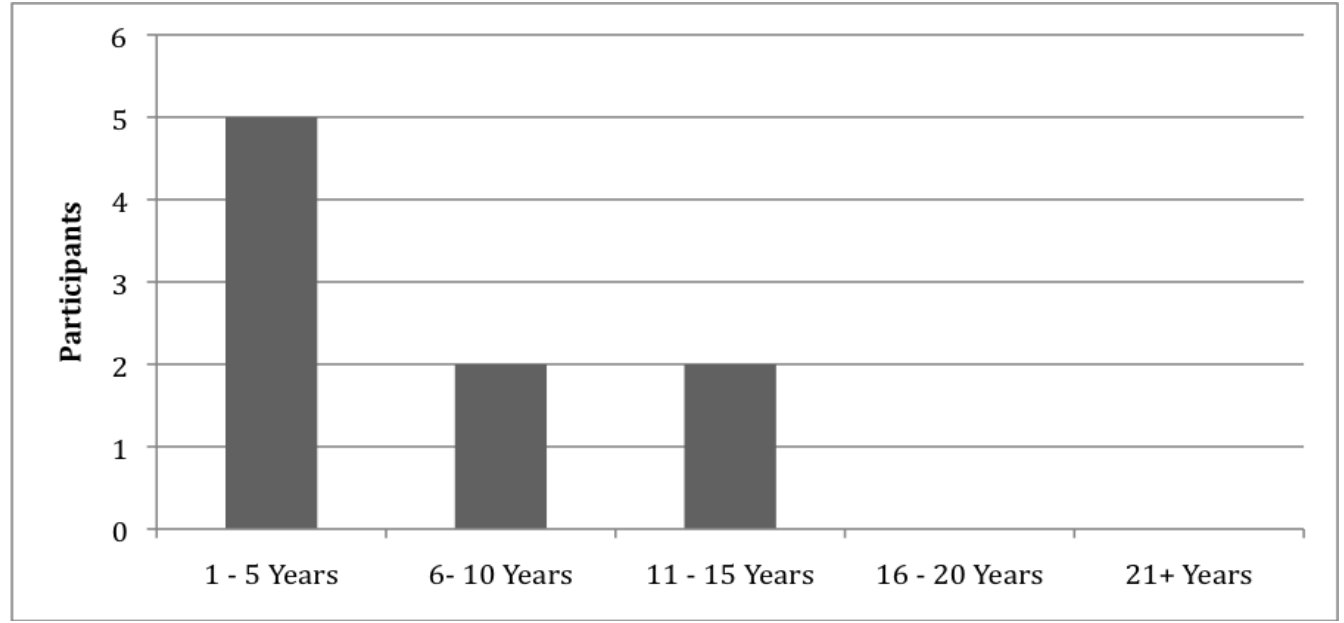

Figure 1. Participant's Years of Technology Architecture Experience

Upon investigation of research question 1, participant responses indicate that the majority use between 7 to 9 foresight methods during the creation of technology blueprints as shown in Figure 2. While this result is slightly higher than Popper [7] findings, it is consistent with variation that he observed during his study.

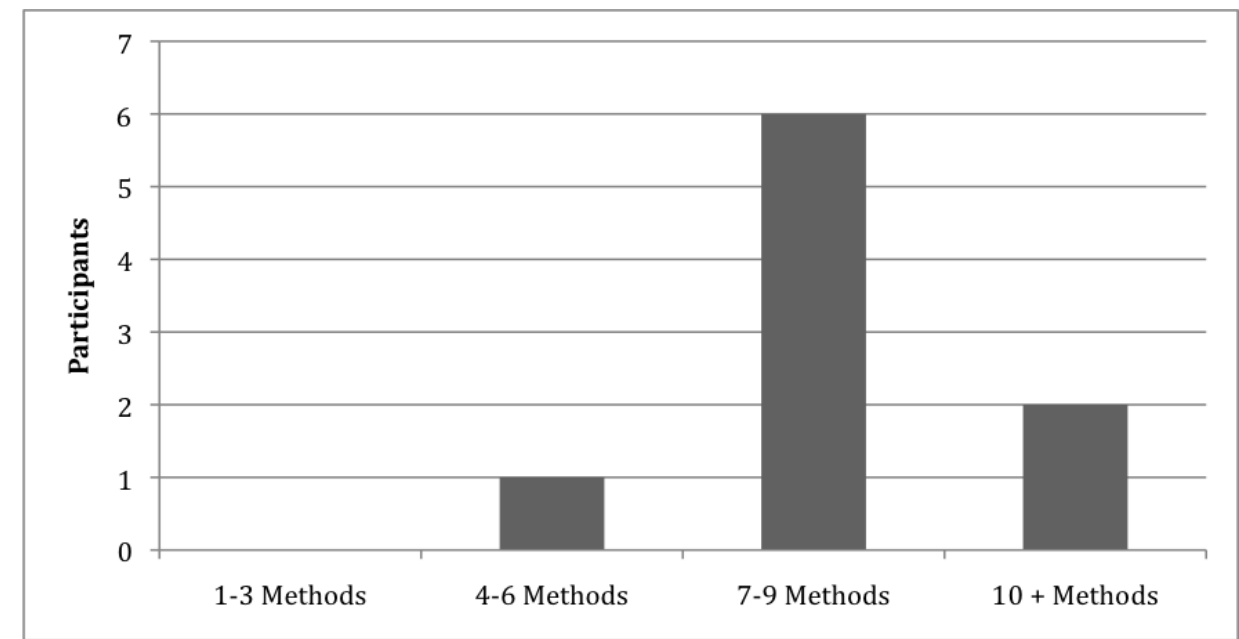

Figure 2. Typical Number of Methods Used In Technology Blueprints

Figure 3 reflects the technology architect's tendency to always use evidence and expertise capabilities during the formation of technology blueprints. This observation addresses research question 2 . This is also consistent with Popper's [7] findings. 


\section{Issues in Information Systems}

Volume 13, Issue 2, pp. 219-225, 2012

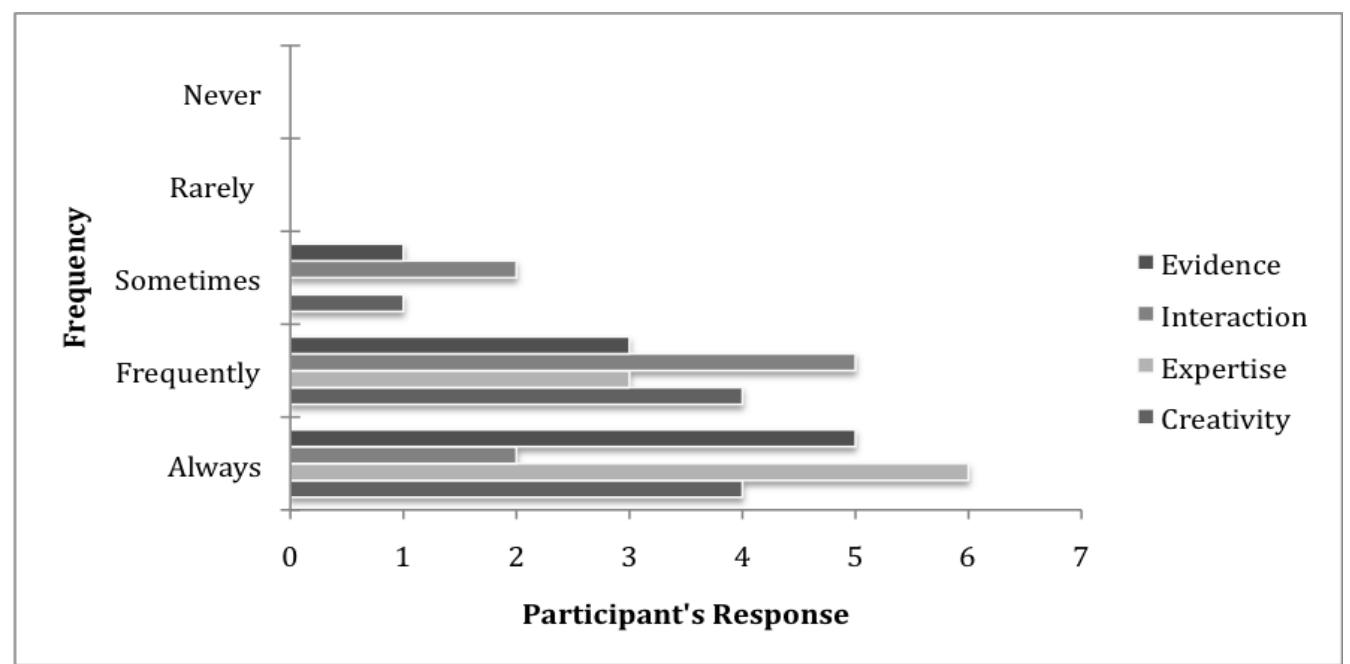

Figure 3. Frequency of Capability Use

This research also surveyed technology architects to identify their decision-making style based on research question 3. According to Kirton [4], the Adaption-Innovation Theory defines and measures a range (on a continuum) of the thinking style that influences all decision-making. Individuals that prefer problem solving in a more detail-oriented manner using established rules and leverage a smaller number of deliberate ideas are more likely to be adaptors. Likewise, individuals that prefer solving problems that demands different perspectives while challenging established guidelines and using a larger number of disparate ideas are more likely to be innovators. Thus, adaptors are concerned with 'doing things better' and innovators are concerned with 'doing things differently'.

The Paradox of Structure describes how one's comfort, or tolerance, with structural boundaries is both enabling and limiting with respect to one's acceptance of change [4]. Additionally, the Paradox of Structure will influence the degree, direction, and speed of that change. Individuals with similar cognitive style (homogenous) would share similar, but not the same, acceptance to the perceived change. Thus, it is reasonable to state that individuals with very different cognitive styles (heterogeneous) could have significant resistance to the perceived change. From this theory, five generic phrases of decision-making were created. These phrases range from a more innovative ("I think outside the box and frequently challenge conventional thinking with different choices") to a more adaptive cognitive style (I only make decisions based on proven experience and documented results"). When selecting foresight capabilities, adaptors would be more likely to use evidence and expertise; innovators would be more likely to select interaction and creativity.

Figure 4 shows their responses to a question that asks for them to select the phrase that most accurately reflects their decision-making choices. 


\section{Issues in Information Systems}

Volume 13, Issue 2, pp. 219-225, 2012

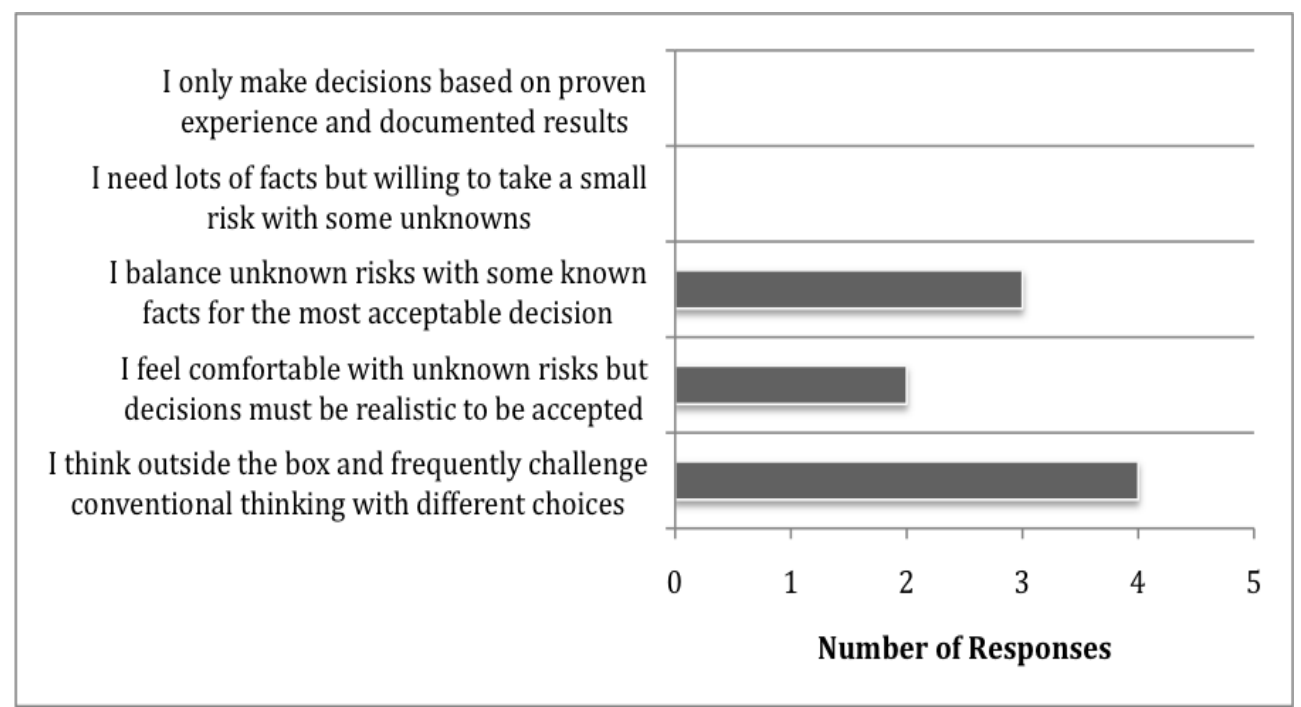

Figure 4. Technology Architect's Decision-Making Personality

The majority of respondents indicated a more innovative style to decision-making. This is an interesting observation because it is not consistent with the frequency of foresight capabilities used. Expertise and evidence capabilities, which are more frequently used, are more adaptive in nature while creativity and interaction are more innovative in nature.

When examining the technology blueprint process of the research participants, it also has a five-phase process as identified by previous studies. The technology blueprints, at this firm, have the following phases:

- Initiation - determine scope, timelines, and key stakeholders

- Baseline - interview experts and knowledgeable stakeholders to identify the current technology use, issues and challenges

- $\quad$ Target - create the vision and future patterns for the use for the identified technology

- Roadmap - identify the core process steps and implementation plans

- Transition - provide ownership models and financial trade-off analysis to migrate threat and maximize opportunities.

As a result, the technology blueprint phases are consistent with previous research and documented foresight activities.

\section{CONCLUSIONS}

In 1991, Millet and Horton [6] in their book, A Manager's Guide to Technology Forecasting, highlights the following observations:

1. no technology forecaster or analyst relies on just one, or even a few methods

2. the variations in methods, techniques and applications typically reflect the topic of analysis and the corporate culture more than the state of the art of the method

3. there appears to be a growing gap between decision-making managers and information-producing analysts.

Even after two decades since their publication, these statements seem to be just as relevant today as in the past.

Based upon the results of this study, this study concluded that technology blueprints seem to have similar attributes to other foresight activities based on capabilities and frequency of foresight methods used. This is significant because it supports the concept that technology blueprints can be used as a technology foresight framework. The 


\section{Issues in Information Systems}

Volume 13, Issue 2, pp. 219-225, 2012

number of methods used during technology blueprints is consistent with other foresight activities. The selection of foresight capabilities is consistent with previous research. Additionally, the number and description of phases are consistent between foresight activities and technology blueprints.

The perceived discrepancy between the preference of the foresight capabilities and the perceived cognitive style of the technology architects requires further exploration. Initial research indicates that technology architects prefer to use methods that are not consistent with technology architect's decision-making style. As Popper [7] indicated, technology architects should explore more use of creativity and interaction capabilities. Perhaps the structure of the process and the corporate culture (as referenced by Millet and Horton) could have a more significant influence on foresight method than previously observed. This study has two significant limitations: sample size and validated questionnaire. Future research should include a larger sample of technology architects employed in diverse firms and industries. Additionally, the validated Kirton Adaption-Innovation (KAI) should be applied to gather a more accurate assessment of the participant's decision-making cognitive style.

Technology blueprints provides a framework to identify trends by relating the associated information under investigation to solutions. By analyzing information sources, technology blueprints allow organizations to position to strategically lead with a plan and/or react with agility in a clear technology direction. By realizing the differences in adaptive and innovative cognitive styles, along with the Paradox of Structure, as it applies to technology blueprints, the most appropriate foresight capability can be leveraged to provide the best opportunity for optimal acceptance. Additional research should also study the relationship between the selection of foresight methods and the technology architect's cognitive style.

\section{REFERENCES}

1. Butler, J. M. (2003). Technology Blueprints: Technology Foundations for High Performance Companies. Aspatore Books. Eagan, MN.

2. Carlson, L. (2004). Using technology foresight to create business value. Research Technology Management. 47(5). Pg. 51-60.

3. Clements, P. and Northrop, L., Software Product Lines: Practices and Patterns. Addison-Wesley, 2002.

4. Kirton, M.J. (2003). Adaption-Innovation: In the Context of Diversity and Change. Routledge.

5. Martino, J., Technological Forecasting for Decision Making, $3^{\text {rd }}$ Edition. McGraw Hill, 1993.

6. Millett, S. and Horton, E. A Manager's Guide to Technology Forecasting and Strategy Analysis Methods. Battelle Press, 1991.

7. Popper, R. (2008). How are foresight methods selected?. Foresight. 10(6). Pg. 62-89.

8. Rohrbeck, R., Arnold, H., and Heuer, J. (2007). Strategic Foresight - a case study on the Deutsche Telekom Laboratories. ISPIM-Asia Conference, 2007. New Delhi, India. Pg. 12.

9. Ross, J. W., Weill, P., Robertson, D. (2006). Enterprise Architecture As Strategy: Creating a Foundation for Business Execution. Harvard Business Press.

10. Smith, J. E., Saritas, O. (2011). Science and technology foresight baker's dozen: a pocket primer of comparative combined foresight methods. Foresight, 12(2). Pg. 79-96.

11. Spewak, S. H. \& Hill, S. C. (1992). Enterprise Architecture Planning - Developing a Blueprint for Data Applications and Technology. John Wiley.

12. Twiss, B., Forecasting for Technologists and Engineers, Peter Peregrinus. 1992, ISBN: 0-86341-265-3.

13. Zgurovskii, M. Z. and Pankratova, N. D. (2003). An information platform for scenario analysis in technology foresight problems. Cybernetics and Systems Analysis. 39(4). Pg. 564 - 575. 